reassured if they understand what is unexpected pain after surgery and have easy access to return to hospital.

I had a benign tumour expertly removed from the spinal canal, requiring a spinal fusion. The anesthesia, surgery and nursing teams were so good that I used the experience to learn how to improve care that I give. I was determined to be a patient and not use medical knowledge or access for special favour. I had not anticipated that I would have to rate my pain out of 10 within moments of waking up from the operation. I considered the matter quickly and broke my vow not to use my knowledge of postoperative care. I answered "zero." Acetaminophen and ibuprofen were sufficient to allow a speedy and enjoyable recovery.

\section{Vivian C. McAlister, MB \\ Coeditor, Canadian fournal of Surgery}

Competing interests: None declared.

DOI: $10.1503 /$ cjs. 017418

\section{References}

1. Phillips DM. JCAHO pain management standards are unveiled. Joint Commission on Accreditation of Healthcare Organizations. $7 A M A$. 2000;284:428-9.

2. Gounder C. Who is responsible for the pain-pill epidemic? The New Yorker. 2013 Nov. 8. Available: www.newyorker.com/business/currency/ who-is-responsible-for-the-pain-pill-epidemic (accessed 2018 Nov. 12).
3. Government of Canada. Debates of the Senate. 2004;142:243-4. Available: https://sencanada.ca/Content/SEN/Chamber/381/Debates/ pdf/013db_2004-11-04-e.pdf (accessed 2018 Nov. 12)

4. Purdue Pharma. National pain awareness week - message from Dr Tom Koutsavlis, vice president, research development, Purdue Pharma (Canada). Available: http://purdue.ca/en/2017/11/06/national-pain -awareness-week-message-from-dr-tom-koutsavlis-vice-president -research-development-purdue-pharma-canada/ (accessed 2018 Nov. 12).

5. Canadian Pain Society. Pain is poorly managed in Canada. Available: https://c.ymcdn.com/sites/www.canadianpainsociety.ca/resource/ resmgr/Docs/pain_fact_sheet_en.pdf (accessed 2018 Nov. 12).

6. Lopez G. The growing number of lawsuits against opioid companies, explained. Vox 2018 May 15. Available: www.vox.com/policy-and -politics/2017/6/7/15724054/opioid-epidemic-lawsuits-purdue-oxycontin (accessed 2018 Nov 12).

7. Former FDA head: Opioid epidemic one of "great mistakes of modern medicine." CBS News 2016 May 9. Available: www.cbsnews.com/news/ former-fda-head-doctor-david-kessler-opioid-epidemic-one-of-great -mistakes-of-modern-medicine/ (accessed 2018 Nov 12).

8. Mitchell A, van Zanten SV, Inglis K, et al. A randomized controlled trial comparing acetaminophen plus ibuprofen versus acetaminophenplus codeine plus caffeine after outpatient general surgery. $7 \mathrm{Am}$ Coll Surg 2008; 206: 472-9.

9. Hartford LB, Van Koughnett JAM, Murphy PB, et al. Standardization of Outpatient Procedure (STOP) Narcotics: a prospective non-inferiority study to reduce opioid use in outpatient general surgical procedures. $\mathcal{F}$ Am Coll Surg. 2018 Sep 25. pii: S1072-7515(18)32038-6. doi: 10.1016/j. jamcollsurg.2018.09.008. [Epub ahead of print].

10. Government of Canada. National Defence and the Canadian Armed Forces. The Road to Mental Readiness (R2MR) program. Available: www.forces.gc.ca/en/caf-community-health-services-r2mr/index.page? (accessed 2018 Nov. 12).

\title{
La tyrannie de la question sur le score de douleur postopératoire
}

Les opinions exprimées dans cet éditorial sont celles de l'auteur et ne représentent pas nécessairement celles de l'Association médicale canadienne ou ses filiales.

ertains chirurgiens s'inquiètent que leurs efforts pour que leurs patients aient une expérience chirurgicale positive soient anéantis à la salle de réveil. Une fois le patient éveillé et extubé, je participe habituellement à son transfert sur la civière. Pendant qu'on étend sur lui une couverture chaude, je lui demande si l'intervention s'est bien déroulée pour lui, en lui confirmant que tout a bien été. Mon but est d'annuler l'effet de la question qui dominera son expérience en salle de réveil : «Sur une échelle de 1 à 10 , où se situe votre douleur? $\gg$ Il ne viendrait à personne du milieu des affaires d'accueillir des clients en les interrogeant sur leur degré d'insatisfaction. Imaginez alors ma réponse lorsque je me suis réveillé après une anesthésie, un visage étranger penché sur moi qui me posait à voix haute la question fatidique.
Je n'ai découvert que récemment pourquoi la question sur « le score de douleur sur une échelle de 1 à $10 »$ a la vie si dure. En 2000, les pressions exercées par l'American Pain Society (APS) ont réussi à faire inclure le score de douleur dans la liste de vérification postopératoire initiale demandée par la commission mixte pour l'agrément des établissements de santé qui décernent leur certificat aux hôpitaux américains ${ }^{1}$. Les médecins qui se spécialisent en gestion de la douleur s'inquiétaient que la douleur soit trop peu traitée; leur campagne a gommé les distinctions entre douleur chronique et douleur postopératoire. L'APS a affirmé que les nouveaux opiacés plus puissants allaient soulager la douleur sans risque de dépendance et nous sommes ainsi passés de la mépéridine à la codéine, puis au fentanyl et à l'oxycodone pour traiter les mêmes problèmes et ce, sans amélioration apparente ${ }^{2}$. 
La Société canadienne de la douleur (SCD) poursuit cette campagne. Des activistes ont fait pression sur le Sénat en 2004 pour que la première semaine de novembre devienne la toute première Semaine nationale de sensibilisation à la douleur. Tous les exemples utilisés pour convaincre les politiciens concernaient des maladies chroniques $^{3}$. Purdue Pharma (Canada) a fait la promotion de la Semaine nationale de sensibilisation à la douleur avec un communiqué de presse qui insistait sur la douleur chronique $^{4}$. Ce communiqué faisait référence à une fiche d'information de la $\mathrm{SCD}^{5}$; on ignore qui a rédigé cette fiche, La douleur au Canada. Les quatre premiers des points qui la composent mettent en lumière l'insatisfaction à l'endroit des soins postopératoires avec les énoncés suivants : «Malgré nos connaissances et les moyens technologiques dont nous disposons, les Canadiens continuent de souffrir après une intervention chirurgicale, et ce, même dans les meilleurs hôpitaux»; « seulement $30 \%$ des médicaments prescrits sont administrés, $50 \%$ des patients ayant des douleurs modérées ou graves ne reçoivent aucun analgésique après une intervention chirurgicale et la situation est loin de s'améliorer »; « après une intervention chirurgicale courante (hernie inguinale, chirurgie mammaire ou thoracique, chirurgie du genou ou de la hanche), la douleur postopératoire aigüe est suivie d'une douleur persistante dans $10 \%$ à $50 \%$ des cas $»^{5}$.

Aux États-Unis et au Canada, les compagnies pharmaceutiques sont passées par les sociétés nationales vouées au traitement de la douleur, les associations d'infirmières et infirmiers et les régulateurs pour promouvoir leur message voulant que les opiacés ne sont pas assez prescrits. Malgré la série de recours en justice qui lui ont été intentés pour ses pratiques de commercialisation et qui lui ont valu des amendes et des ententes totalisant plus de 600 millions de dollars ${ }^{6}$, l'industrie continue de profiter des superopioïdes à longue action comme l'OxyContin, qui lui rapportent environ 30 milliards de dollars par année. Le $\mathrm{D}^{\mathrm{r}}$ David Kessler était commissaire à la Food and Drug Administration (FDA) lorsque OxyContin a été approuvé; il croit aujourd'hui que cette approbation a été une erreur ${ }^{7}$. C'est donc avec nervosité que nous avons accueilli l'approbation du sufentanil sublingual, un médicament 500 fois plus puissant que la morphine.

Dans le contexte de l'actuelle crise des opioïdes, de nombreux exemples de dépendance sont attribués à leur utilisation en période postopératoire ou post-traumatique qui devait être de courte durée. N'oublions pas que les opioïdes prescrits et non utilisés sont aussi considérés comme une source significative d'accès inapproprié. Les chirurgiens n'ignorent pas le problème. Plusieurs études se sont penchées sur les façons de limiter l'utilisation post- opératoire des opioïdes. Mitchell et ses collaborateurs ${ }^{8}$ de Halifax ont montré qu'un anti-inflammatoire non stéroïdien (AINS) est équivalent à la codéine lorsqu'il est utilisé avec l'acétaminophène après une chirurgie générale. Hartford et ses collaborateurs ${ }^{9}$ de London ont de leur côté encouragé leurs patients à prendre de l'acétaminophène et un AINS après une chirurgie d'un jour, en leur remettant une ordonnance de tramadol de courte durée à faire exécuter en cas de douleur intercurrente. Moins de $30 \%$ des patients ont fait exécuter leur ordonnance, et ils sont nombreux à n'avoir utilisé que quelques comprimés. Ces études suggèrent fortement que la surprescription des opioïdes après une chirurgie constitue un problème.

Nous avons des leçons à tirer de l'expérience militaire. Le programme En route vers la préparation mentale des Forces armées canadiennes est conçu pour développer la résilience face au stress opérationnel en anticipant les conditions du déploiement et en contrôlant les attentes ${ }^{10}$. Nous pourrions adapter ce programme pour préparer nos patients à la chirurgie. L'absence totale de douleur postopératoire n'est pas une attente réaliste. À la place, il faut comprendre l'inconfort prévisible et reconnaître les mesures qui permettront aux patients de se rétablir en utilisant au minimum les opioïdes. Parfois superflues, les restrictions concernant l'exercice ou le lever donnent une impression de fragilité et provoquent de l'anxiété. Il est possible de rassurer les patients en leur expliquant ce que serait une douleur postopératoire anormale et en facilitant leur retour à l'hôpital en cas de besoin.

Des mains expertes m'ont retiré une tumeur bénigne au canal rachidien qui a nécessité une fusion des vertèbres. Les équipes d'anesthésie, de chirurgie et de soins infirmiers ont été si compétentes que j'ai voulu utiliser cette expérience pour améliorer les soins que je prodigue. J'ai fait vœu d'être un simple patient, de ne pas utiliser mes connaissances médicales et bien sûr de ne pas demander de traitement de faveur. Je n'avais pas prévu que j'aurais à classer ma douleur sur une échelle de 1 à 10 en salle de réveil quelques minutes après mon opération. J'ai réfléchi rapidement et j'ai brisé ma promesse de ne pas utiliser mes connaissances médicales en période postopératoire. J'ai répondu « zéro ». L'acétaminophène et l'ibuprofène ont suffi pour que je me rétablisse rapidement et agréablement.

\section{Vivian C. McAlister, MB \\ Corédacteur en chef, fournal canadien de chirurgie}

Intérêts concurrents : Aucun déclaré.

DOI: $10.1503 /$ cjs.017618

Références : voir la version anglaise à la page précédente ou en ligne au http://canjsurg.ca/vol61-issue6/61-6-364/. 